\title{
MS089.003
}

\section{Mullite - towards a unified superspace model}

Paul Benjamin Klar ${ }^{1}$, Noelia de la Pinta1 ${ }^{1}$, Gabriel Alejandro Lopez ${ }^{2}$, Iñigo Etxebarria ${ }^{2}$, Tomasz Brezcewski ${ }^{2}$, Gotzon Madariaga ${ }^{1}$

${ }^{1}$ Dpto de Física de la Materia Condensada, Facultad de Ciencia y Tecnología, Universidad del País Vasco UPV/EHU, Bilbao, Spain, ${ }^{2}$ Dpto de Física Aplicada II, Facultad de Ciencia y Tecnología, Universidad del País Vasco UPV/EHU, Bilbao, Spain E-mail: paulbenjamin.klar@ehu.eus

Mullite $\mathrm{Al}_{4+2} \mathrm{Si}_{2-2} \mathrm{O}_{10-x} \square_{x}$ ( $\square=$ vacancy) is a well known material in the ceramics industry with a broad range of applications, but from a fundamental point of view its crystal structure is not completely understood. The dependence of the modulation wave vector $\mathrm{q}=(\mathrm{a} 0 \mathrm{y})$ on the composition lacks an explanation and it is not known why for vacancy concentrations $\mathrm{x}>0.5$ a lowering of the symmetry is observed [1].

A recent superspace model (SSM) described in Pbam(a01/2)0ss results in a brick pattern of alternating vacancy blocks and vacancy-free blocks [2]. Assuming that, independent of the composition, the brick pattern is maintained and intermeshing of vacancy blocks is avoided by the structure, the relation $a=(1-x) / 2$ is derived from the SSM. It allows to predict the vacancy distribution of any mullite with $x \leq 0.5$ including the cases of the prominent $3 / 2$ mullite $(x=0.25), 2 / 1$ mullite $(x=$ $0.4)$ and $5 / 2$ mullite $(x=0.5)$. Models derived from the SSM are depicted in the Figure below. The limit results from one of the atomic domains which has a negative length for $x>0.5$, i.e. a new SSM is required. This border coincides with an observed change of the symmetry from orthorhombic to monoclinic because $y$ then deviates from $1 / 2$. As the deviation is very small, the nature of the modulation seems to be the same for the complete solid solution range and the dependence of $\mathrm{q}$ on the composition is a result from the block pattern that determines the vacancy distribution. The SSM can be easily adapted to monoclinic symmetry with superspace group $\mathrm{P} 2{ }_{1} / \mathrm{a}(\mathrm{a} 0 \mathrm{y}) 0$ s to describe a monoclinic brick pattern of alternating vacancy blocks and vacancy-free blocks. These predictions are strongly supported by HRTEM observations and image simulations [1].

However, the suggested vacancy ordering scheme is expected to be only present when higher order satellite reflections are present in the reciprocal space. In many samples this is not the case and first order satellite reflections appear as maxima in a characteristic diffuse scattering pattern [3], i.e. that the long-range vacancy ordering is not present and vacancies are rather mid-range ordered. Nevertheless the dependency of $\mathrm{q}$ on $\mathrm{x}$ is maintained independent of the diffuseness, which indicates that the disordered vacancy distribution is strongly related to the structure of the highly ordered SSM. In [2] it is also shown that a disordered SSM of [3] and the ordered SSM are indeed strongly related. It means that although mullite samples usually exhibit a lower ordering degree than described by the model, it establishes a basic vacancy ordering pattern from which structural models that take diffuse scattering into account must originate.

This work is supported by the Basque Government (No. ПT-779-13 \& predoctoral grant) and FEDER (MAT2015-66441-P). A travel grant by the Master and Doctoral School (UPV/EHU) is highly appreciated.

[1] Ylä-Jääski, J. \& Nissen, H.-U. (1983). Phys. Chem. Miner., 10, 47-54.

[2] Klar, P. et al. (2017). Acta Cryst. B, accepted.

[3] Birkenstock, J. et al. (2015). Acta Cryst. B71, 358-368.

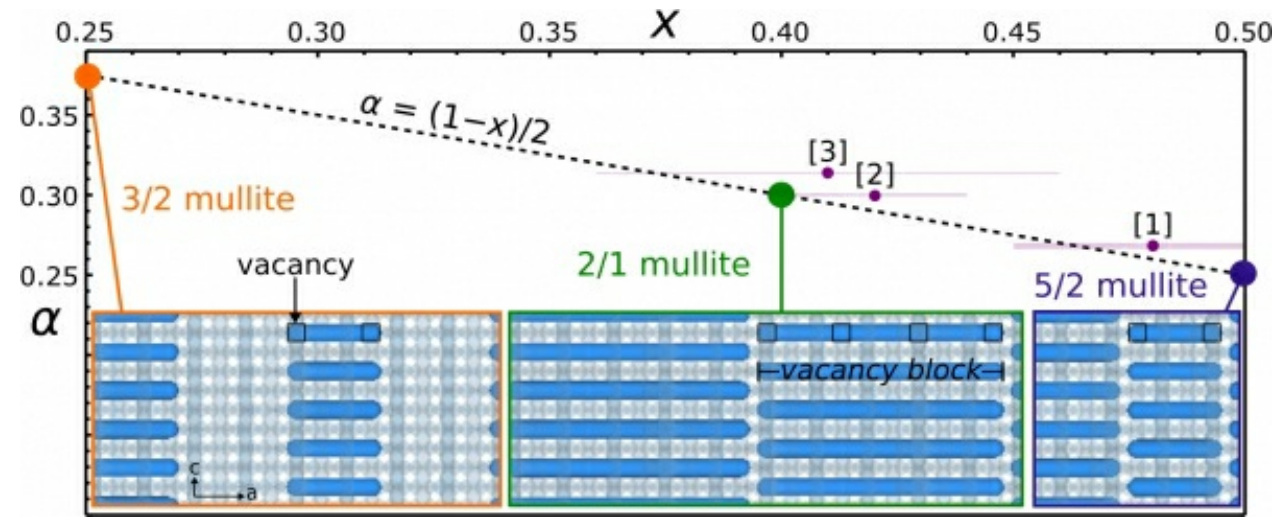

Keywords: vacancy ordering, modulated crystal structure, mullite 